\title{
Differential Expression of miRNAs in Sorghum bicolor under Drought and Salt Stress
}

\author{
Rania S. El Sanousi ${ }^{1}$, Nada B. Hamza ${ }^{2 *}$, Awadalla A. Abdelmula1 ${ }^{1}$, Ismail A. Mohammed ${ }^{1}$, \\ Seif M. Gasim', Neeti Sanan-Mishra ${ }^{3}$ \\ ${ }^{1}$ Faculty of Agriculture, University of Khartoum, Khartoum North, Sudan \\ ${ }^{2}$ Commission for Biotechnology \& Genetic Engineering National Centre for Research, Khartoum, Sudan \\ ${ }^{3}$ Plant Molecular Biology Group, International Centre for Genetic Engineering and Biotechnology, ArunaAsaf Ali \\ Marg, New Delhi, India \\ Email: "nada.hamza@gmail.com
}

Received 23 February 2016; accepted 22 April 2016; published 25 April 2016

Copyright (C) 2016 by authors and Scientific Research Publishing Inc.

This work is licensed under the Creative Commons Attribution International License (CC BY). http://creativecommons.org/licenses/by/4.0/

\section{c) (i) Open Access}

\begin{abstract}
The regulatory mechanisms of drought and salt-associated miRNAs have not been fully understood in Sorghum bicolor. In this study, we investigated the effect of salinity stress $(200$ and 300 $\mathrm{mM} \mathrm{NaCl}$ ) and drought stress at pre- and post-flowering stages on the expression pattern of small regulatory RNAs in six Sorghum genotypes using semi-quantitative reverse transcriptase PCR (RTqPCR). The results indicated that both drought and salt stresses altered the expression pattern of miRNAs in a dose-dependent manner. However, each miRNA responded to drought and salt stress in a different pattern among the six sorghum genotypes. miR156, miR167, miR168 and miR399 give different expressions levels compared to other studied miRNAs which may attribute to the adaption of sorghum to drought and salt stress and are good candidates for improving sorghum by transgenic technology.
\end{abstract}

Keywords

miRNA, Up-Regulation, Down-Regulation, Pre-Flowering, Post-Flowering

\section{Introduction}

MicroRNAs are endogenous, non-coding RNA molecules, 21 - 24 nucleotides in length playing crucial roles in

"Corresponding author.

How to cite this paper: El Sanousi, R.S., Hamza, N.B., Abdelmula, A.A., Mohammed, I.A., Gasim, S.M. and Sanan-Mishra, N. (2016) Differential Expression of miRNAs in Sorghum bicolor under Drought and Salt Stress. American Journal of Plant Sciences, 7, 870-878. http://dx.doi.org/10.4236/ajps.2016.76082 
the regulation of gene expression at the post-transcriptional level in eukaryotes and viruses [1] [2]. These regulatory small RNA molecules achieve their roles through sequence-specific interactions with complementary sites on target mRNA that leads to their degradation or translational repression [3]. Genomic studies have shown the involvement of plant miRNAs in many developmental processes such as organ development, root development, seeds germination, flowering initiation, seed production and in diverse stress responses [4].

Drought and salinity are two of the most serious problems for crop production worldwide. For agricultural and environmental sustainability, plants need to develop an efficient strategy such as modulation of gene expression to enhance stress tolerance. Plants use adaptive responses operating at the post-translational levels to cope with these environmental challenges. Nineteen sorghum genotypes from Sudan were studied by [5] under drought condition, their results showed significant differences among genotypes, where differential yield response was reported. Several studies have demonstrated that miRNAs play important roles in the responses to biotic and abiotic stimulus. Expression of plant miRNAs has been detected as up- and down-regulated levels up on treatment of diverse stress conditions [6]. Some of the genes related to stress response are post-transcriptionally regulated by miRNAs, such as NFYA5 transcription factor, which promotes drought resistance in Arabidopsis [6]. Recent studies report that miR393 is commonly and consistently up-regulated during drought stress in many plants such as Arabidopsis, Medicago, common bean, and rice [7]. Also miR398, which is induced by oxidative stress, such as salinity, drought, and extreme temperatures, regulates two $\mathrm{Cu} / \mathrm{Zn}$ superoxide dismutases, under drought conditions. Numerous methodologies have been developed for rapid, sensitive, specific and genomewide detection of miRNAs. One of these methods is semi-quantitative reverse transcriptase PCR (q RT-PCR) [8].

Sorghum is the fifth most important cereal crop in the world after wheat, rice, maize and barley and it is an economically important cereal plant in Asia and Africa; it feeds more than 500 million people in 98 countries, especially in arid and semi-arid regions [9]. Sorghum is genetically suited to hot and dry conditions and is known for its ability to grow in harsh environments. Its relatively small diploid genome (735 Mbp) and C4 plant physiology are among the numerous mechanisms that allow it to survive and be productive under these conditions [10]. Different groups have identified several microRNAs (miRNAs) in sorghum [11]-[13]. The deciphering of miR expression patterns under abiotic stress in different cultivars of sorghum will provide a better understanding of their genetic responses. In an earlier study, we investigated the effect of drought stress on the expression levels of selected miRNAs across 12 elite accessions of African sorghum (REF). A correlation was drawn with their predicted targets to obtain meaningful insights into the differential response of the miRNAs in the varied genotypes. In this work we have studied the involvement of miRNAs in response to drought and salt stresses in sorghum using semi-quantitative (Reverse Transcription) RT-PCR technology.

\section{Materials and Methods}

The materials studied consisted of six sorghum cultivars (Sorghum bicolor (L.) Moench), namely, Wad Ahmed, Tabat, Tetron, Dwarf White Milo, Arfa-Gadamak and Red-Mugud. Seeds of these varieties were obtained from the Gene Bank of the Agricultural Plant Genetic Resources Conservation and Research Center (Agricultural Research Corporation, Wad Medani, Sudan).

\subsection{Induction of Drought Stress}

Seeds were sown on $20^{\text {th }}$ June2014, at the Experimental Farm, Faculty of Agriculture, University of Khartoum, Shambat (Lat. $15^{\circ} 35$ N.; Long. $32^{\circ} 31$ E.). Plants were irrigated immediately after sowing for crop establishment and until the plant size was uniform (35 - 40 days).The experiment was laid out in a split plot with three replicates. Two types of drought stress were conducted, the first type of drought stress was applied after 35 days of sowing (pre-flowering stress) by withholding water from the stressed plots for three weeks then irrigation was continued regularly until end of season. The second type of drought stress was applied after 60 days of sowing (post-flowering stress) by withholding water from stressed plots until end of season. Data analysis was carried out using SPSS 22 program and the means compared by Tukey's multiple range test $(P<0.05)$. Leaves samples were collected from drought stressed plants for RNA isolation.

\subsection{Induction of Salt Stress}

To study the effect of salinity on six sorghum genotypes, a controlled experiment was conducted in growth 
chamber at $28^{\circ} \mathrm{C}$. Treatments were three salinity levels, i.e. control (without $\mathrm{NaCl}$ ), $200 \mathrm{mM}$ and $300 \mathrm{mM} \mathrm{NaCl}$ concentrations. The experiment was arranged in a completely randomized design with three replicates. Data were submitted to analysis of variance (ANOVA) and the means were compared using Tukey's multiple range test $(P<0.05)$. Leaves samples were collected from salt stressed plants for RNA isolation.

\subsection{RNA Isolation and Semi-Quantitative RT-PCR Analysis}

Total RNA was extracted using miRNeasy Mini Kit (Qiagen) according to the manufacturers' instructions, which allows also precipitation of the small RNA fraction (18 - $24 \mathrm{nt})$. miRNA quality was verified on 1.5\% agarose gel followed quantification in a NanoDrop (Q 5000, Japan). Five micrograms of total RNA were used for cDNA synthesis using QuantiMir RT Kit (System Biosciences SBI, Japan) following the user manual. CDNAs were used as templates to determine expression levels of miRNAs and target genes with semi quantitative reverse transcriptase PCR. The amplification was performed using $1 \mu \mathrm{l}$ of the resulting cDNA added to 20 $\mu \mathrm{l}$ PCR reaction mixture GoTaq Green Master Mix (Promega) and amplified with miRNA specific forward primers and universal Reverse Primer as shown in Table 1. The reaction conditions were standardized were as follows: heat denature at $95^{\circ} \mathrm{C}$ for $10 \mathrm{~min}, 30$ cycles of $95^{\circ} \mathrm{C}$ for $15 \mathrm{sec}, 60^{\circ} \mathrm{C}$ for $1 \mathrm{~min}$ and hold at $15^{\circ} \mathrm{C}$. U6 was also amplified and used as an internal control because it is demonstrated uniform expression stability across very different biological backgrounds. Finally $5 \mu \mathrm{l}$ of PCR product was run on 10X polyacrylamide gel electrophoresis in 5X TBE buffer for two hours. After electrophoretic the expression rate of the target miRNAs (normalization) were assessed by measuring the intensity of the bands corresponding to the generated amplicon and compared with the internal control (U6) using Image J program.

\section{Results}

\subsection{Expression Profiles under Drought Stress}

The expression levels of eleven miRNAs were validated in the six sorghum genotypes under pre-flowering drought, post-flowering drought stress and salt stress (200 - $300 \mathrm{mM} \mathrm{NaCl})$. The miRNAs were selected for this study, included sbi-miR156(a/h/i/d), sbi-miR159(a), sbi-miR167(c/f/g), sbi-miR168, sbi-miR393(a/b), sbi-miR160(f), sbi-miR166(a-d), sbi-miR166(f), sbi-miR171(a/b/d/k/i), sbi-miR399(b) and sbi-miR1435(b). A majority of these miRNAs have been reported to play a role under stress conditions in model plant species. All the eleven tested miRNAs were expressed in sorghum genotypes, but their expression level varied from each

\begin{tabular}{ccc}
\hline \multicolumn{2}{c}{ Table 1. Sequences of primers used for semi-quantitative RT-PCR. } \\
\hline miR Names & Forward primer sequences (5' $\rightarrow 3^{\prime}$ ) & bp \\
\hline miR156d & TGACAGAAGAGAGAGAGCACA & 21 \\
miR156 a,h,i & TGACAGAAGAGAGTGAGCAC & 20 \\
miR 159a & TTTGGATTGAAGGGAGCTCTG & 21 \\
miR 167c,g,f & TGAAGCTGCCAGCATGATCTG & 21 \\
miR168 & TCGCTTGGTGCAGATCGGGAC & 21 \\
miR393a,b & TCCAAAGGGATCGCATTGATC & 21 \\
miR160f & TGCCTGGCTCCCTGAATGCCA & 21 \\
miR166a-d & TCGGACCAGGCTTCATTCCC & 20 \\
miR166f & TCGGACCAGGCTTCATTCCTC & 21 \\
miR171 & TGATTGAGCCGTGCCAATATC & 21 \\
miR399b & TGCCAAAGGAGAGCTGCCCTG & 21 \\
miR1435b & AAAATAACTCTACGAATTGAATGG & 24 \\
U6 & CGCAAGGATGACACGCAAATTC & 25 \\
\hline
\end{tabular}


other among the genotypes with some of these miRNAs showing up-regulation and the others down-regulation under abiotic stress. The results of the pre-flowering drought stress showed that sbi-miR166 and sbi-miR1435 were up-regulated while sbi-miR156, sbi-miR159 and sbi-miR393 displayed down- regulated expression level in the six sorghum genotypes (Figure 1). The expression levels of sbi-miR171 and sbi-miR399 were also up-regulated in most genotypes with exception in the genotypes Tabat and Milo, respectively while sbi-miR167 levels were down-regulated in most varieties except Wad-Ahmed (Figure 1). Not much variation was seen in the levels of sbi-miR160 in most varieties except for an up-regulation observed in Tabat. The levels of sbi-miR168 showed a variety specific behavior as it was down-regulated in Tabat, Milo, Tetron and Wad-ahmed varieties but up-regulated in Red-Mugud and Arfa-Gadamak after pre-flowering drought stress (Figure 1). Most miRs showed a drastic deregulation in the Tabat variety. The post-flowering drought stress also influenced the expression profiles of the miRNAs to different levels among the six sorghum genotypes (Figure 2). Generally the higher expression levels of the most miRNAs were represented by the genotype Milo. On the other hand the lowest expression levels of miRNAs were detected in the genotype Tabat. The genotype Tetron showed moderate expression levels of some miRNAs. Also the results showed the up-regulation expression of the eleven miRNAs in different levels among the sorghum genotypes as shown in (Figure 2) On the other hand the results revealed that miR159 and miR168 were slightly up-regulated, while miR166 and miR399 were very low upregulated in sorghum genotypes. Furthermore, miR156, miR393, and miR167 were moderate up-regulated in genotypes.

Our results of pre-flowering and post-flowering drought stress revealed the differential expression pattern among the six sorghum genotypes. The differences were prominent under pre-flowering than in post-flowering stress indicating that pre-flowering stages were highly sensitive to the stress conditions. In Wad Ahmed, which is a drought tolerant phenotype, sbi-miR167, sbi-miR171, sbi-miR1435, and sbi-miR399 were up-regulated during pre-flowering drought treatment. On the other hand in case of the genotype Tabat which described as susceptible phenotype, sbi-miR167 and sbi-miR171 showed an opposite expression pattern under pre-flowering drought stress. In other sorghum genotypes, Red-Mugud, Arfa-Gadamak and Milo which show moderate degree of tolerance to the two types of drought treatments the miR expression patterns were similar to that of Wad-Ahmed.

\subsection{Expression Profiles under Salt Stress}

The results of salt stress exhibited different expression levels of the eleven miRNAs among the six sorghum genotypes. The eleven miRNAs were down-regulated in response to $200 \mathrm{mM} \mathrm{NaCl}$ treatment in the six sorghum genotypes; however sbi-miR156, sbi-miR166, sbi-miR167, and miR171 were up regulated in Arfa-Gadamak and Wad-Ahmed genotypes. Sbi-miR168 showed high, up-regulation in the genotype Red-Mugud while sbimiR393 and sbi-miR399 were up-regulated in the genotypes and Wad Ahmed, respectively (Figure 3). The expression profiles of sbi-miRs 399, 156 and 159 could be correlated under the salt and drought stresses in the Wad-Ahmed genotype. On the other hand obtained results showed down regulation expression of the twelve tested miRNAs among the six sorghum genotypes which exposed to $300 \mathrm{mM} \mathrm{NaCl}$ (Figure 4).

\section{Discussion}

Huge numbers of miRNAs have been identified as stress-responsive in several plant species. Up-regulation of these miRNAs has been observed in diverse plant species in response to drought stress [4]. In a recent study, [14] studied the expression profiling of 8 abiotic stress deregulated miRNAs for 11 sorghum genotypes (grain and forage genotypes) from Sudan under watered and drought stress. Significant deregulation was reported with miR396, miR393, miR397-5p, miR166, miR167 and miR168. Forage accessions (N98 and Atlas) had different expression with miR397-5p. The findings of [14] could be a good indicator of the prevailing drought tolerance mechanism present in the studied sorghum genotypes. Previously, [15] found that miR156, miR166, and miR171 were differentially expressed upon dehydration in barley genotypes. On the other hand miR167, miR168 and miR171 were found to be drought stress responsive miRNAs in Arabidopsis [16]. Consistent with these results, our results here support that the up-regulation of these miRNAs might involve in the adaptive response to drought stress in sorghum genotypes.

On the other hand, obtained results indicated that sorghum genotypes were more sensitive to the high levels of salt concentrations and this might agree with different previously morphological studies. There were significant reductions in sorghum growth reported by [17] when salinity was applied in range from 50 to $150 \mathrm{mM}$. Several 

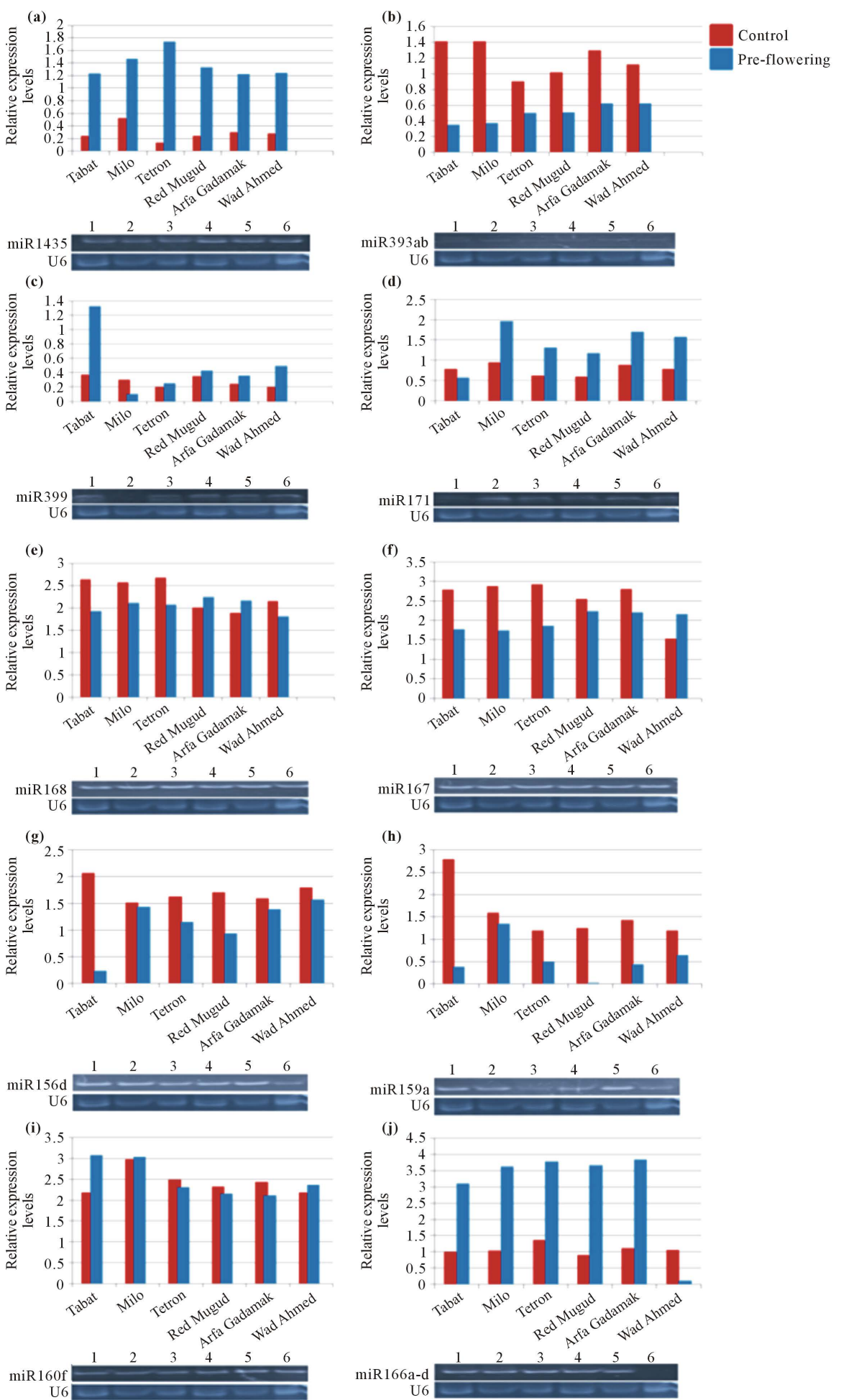

Figure 1. (a): Up-regulation of miR1435 in the six sorghum genotypes. (b): Down-regulation of miR393 in the six sorghum genotypes. (c): Up-regulation of miR399 in the six sorghum genotypes except the genotype Milo. (d): Up-regulation of miR171 in the six sorghum genotypes except the genotype Tabat. (e): Down-regulation of miR168 in the six sorghum genotypes except the genotypes Arfa-Gadamak and Red Mugud. (f): Down-regulation of miR167 in the six sorghum genotypes except the genotype Wad Ahmed. (g): High expression of miR156 d in the genotype Wad Ahmed and low expression in genotype Tabat. (h): Down-regulation of miR159 in the six sorghum genotypes. (i): Up-regulation of miR160f in the genotypes Tabat, Milo and Wad Ahmed. (j): Up-regulation of miR166a-d in all genotypes except in genotype Wad Ahmed. 

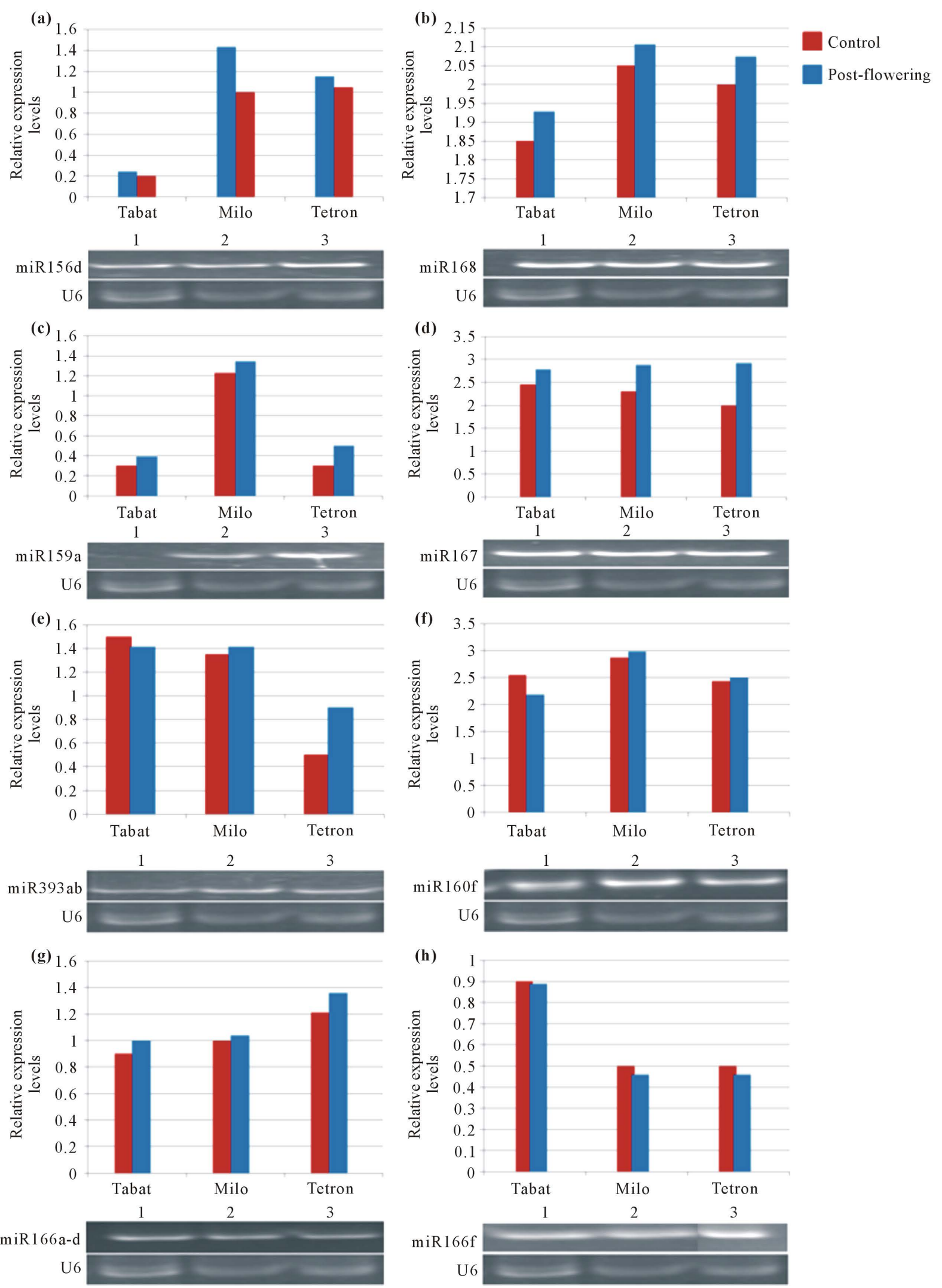

Figure 2. Up-regulation of miRNAs in sorghum genotypes exposed to post-flowering drought stress. 

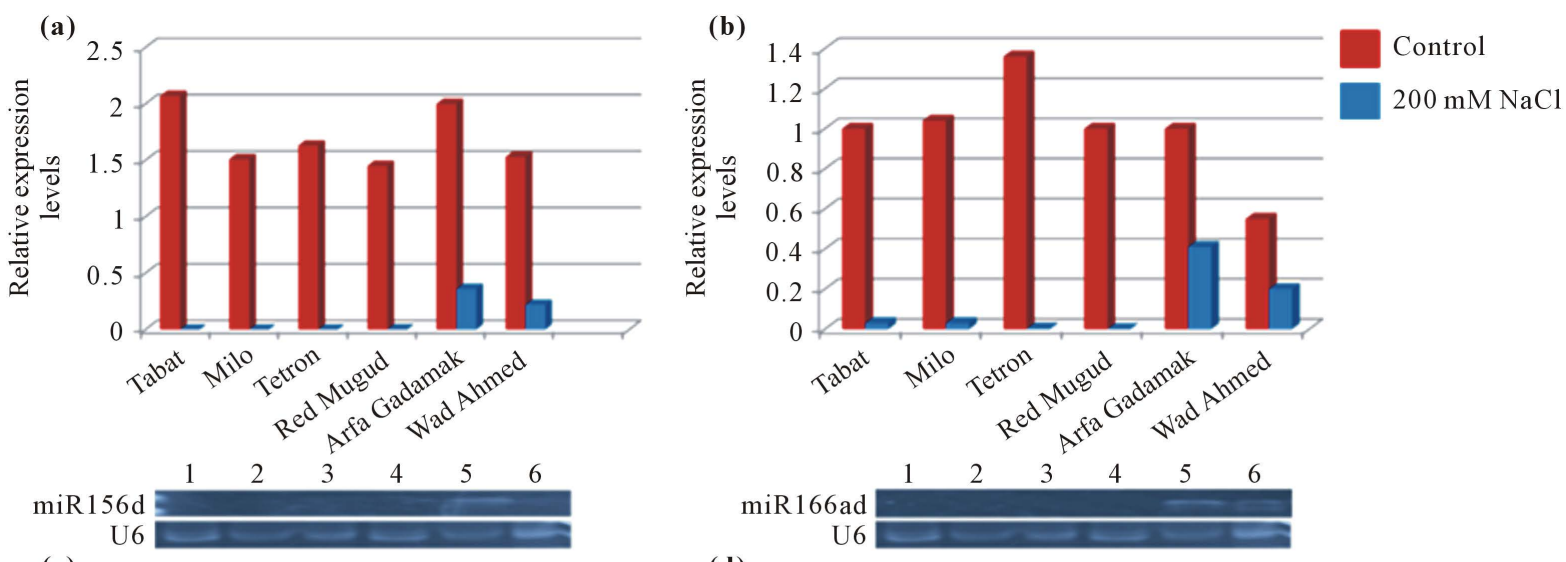

(c)

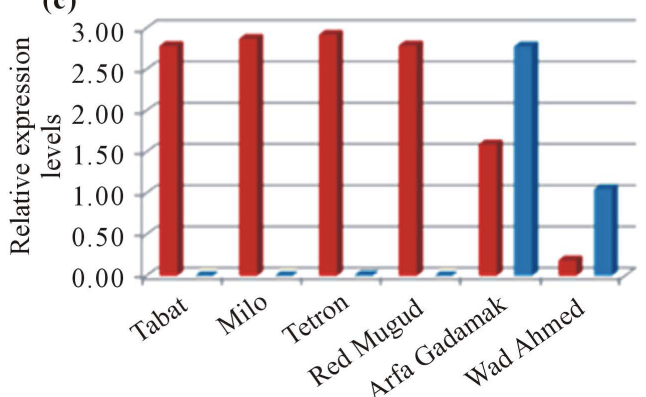

(d)

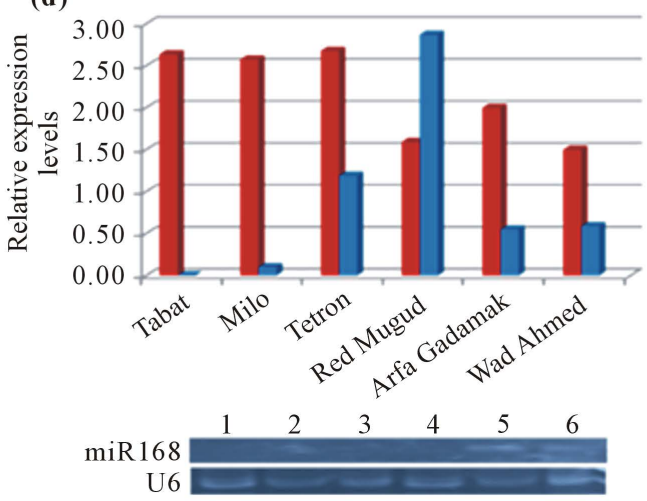

(e)

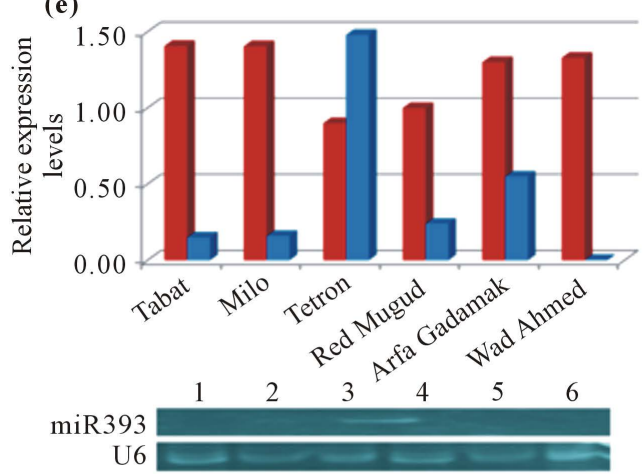

(f)

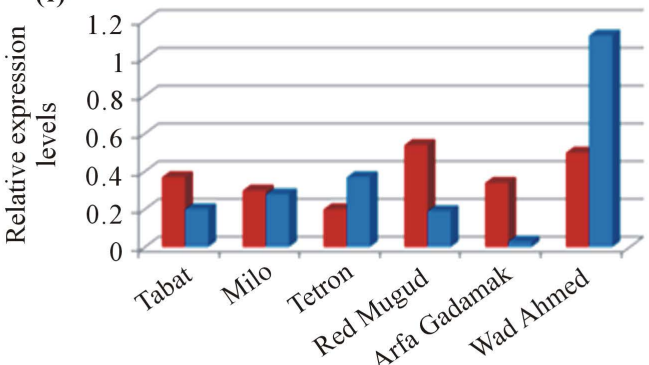

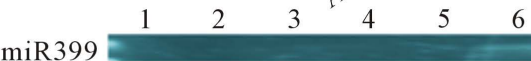
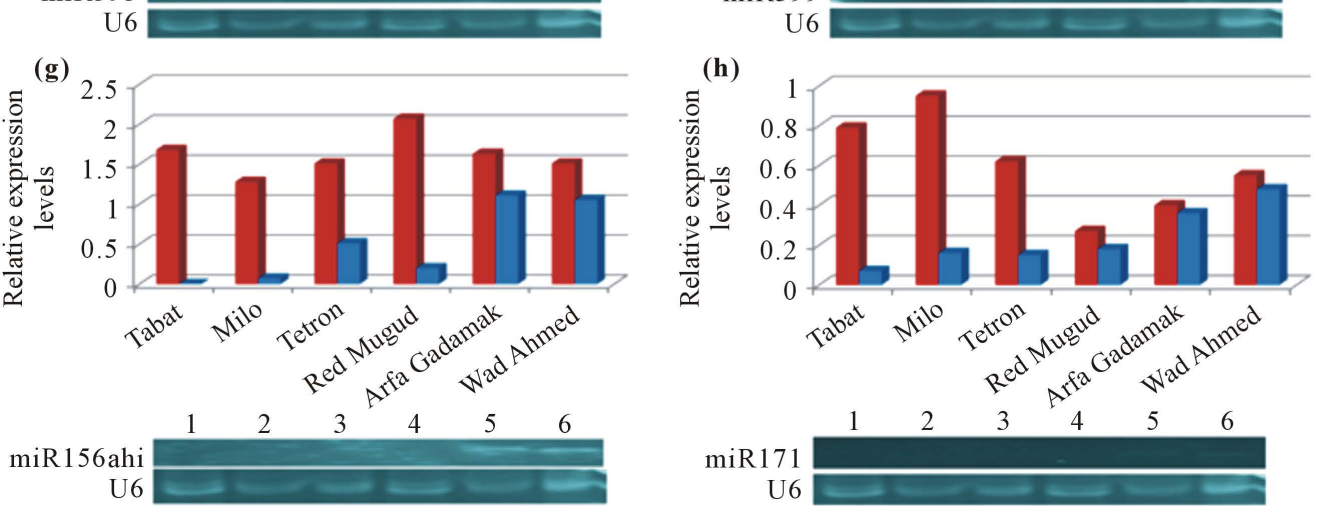

Figure 3. The expression levels of miRNAs in $200 \mathrm{mM}$ salt stress. (a) and (b): Down-regulation of miR156d and miR166ad in all sorghum genotypes. (c): Down-regulation of miR167 in sorghum genotypes except in the genotypes Wad Ahmed and Arfa-Gadamak. (d): Down-regulation of miR168 in sorghum genotypes except in the genotype Red Mugud. (e) and (f): Up-regulation of miR393 and miR399 in genotypes Tetron and Wad Ahmed respectively. (g) and (h): Down-regulation of miR156 and miR171 in all genotypes. 

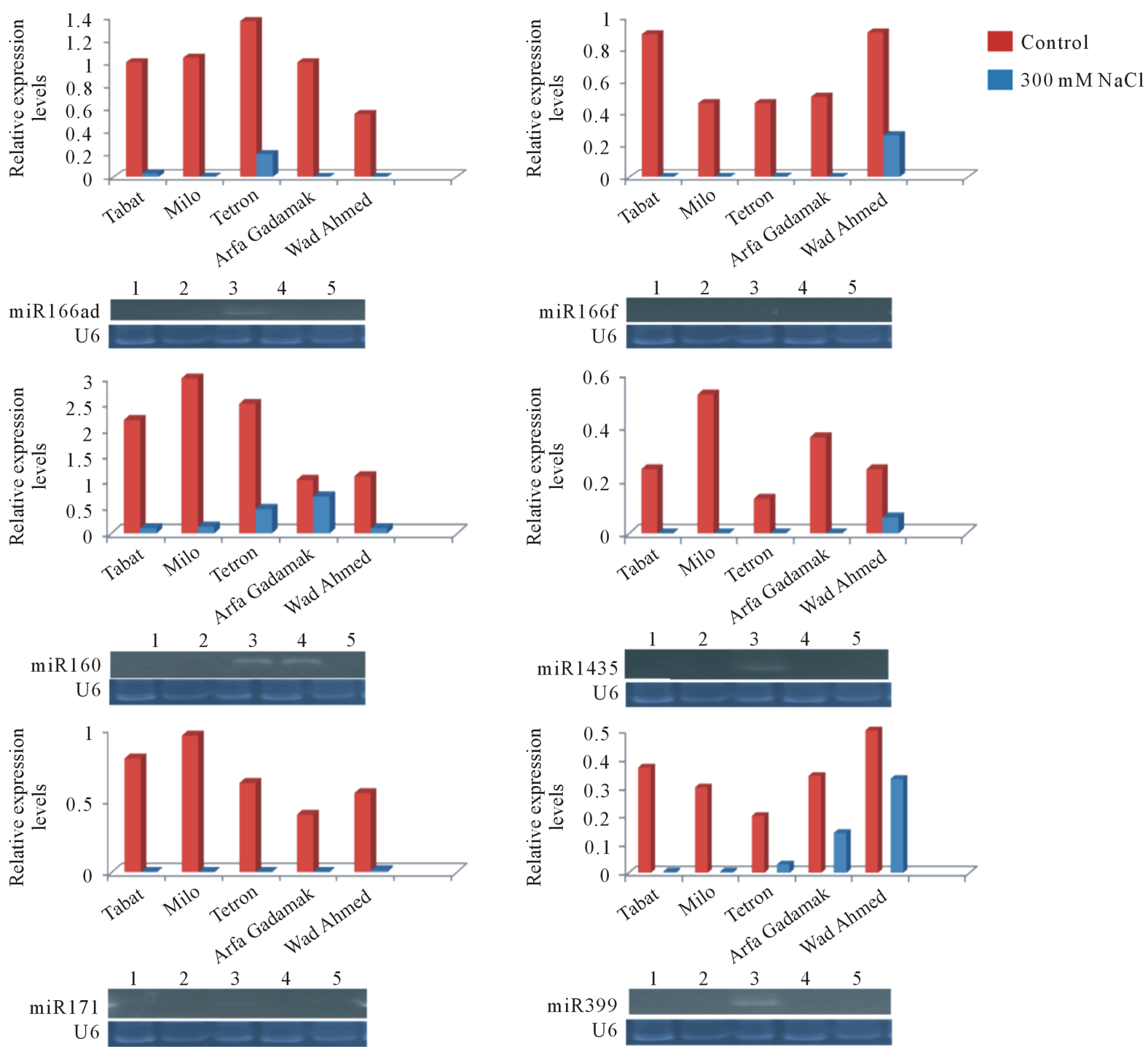

Figure 4. Down-regulation of the studied miRNAs, in the six sorghum genotypes under $300 \mathrm{mM}$ salt stress.

differentially regulated miRNAs have been identified in salt-stressed plants. The results found that miR156, miR156ahi, miR159, miR171, 166ad and miR1435 were down-regulated, whereas miR168, miR167 miR393, miR166, miR399 and miR160f were up-regulated in $200 \mathrm{mM}$ salt-stressed sorghum genotypes. Study presented by [18] found that the expression level of miR156 and miR159 was down-regulated in French bean under salt stress, in contrast, the expression level of miR399 was up regulated. In Arabidopsis, miR156, miR167, miR168, miR171 and miR393 were up regulated in response to salt stress [16]. In conclusion, the present study showed that miRNAs are indeed involved in the plants response to drought and salt stresses as indicated by the number of differentially expressed miRNAs. Also the study showed that each miRNA had its unique mechanism of action during stress response largely dependent on the stress type whereas the high expression was shown under drought stress. Furthermore, some miRNAs such as miR156, miR167, miR168, and miR399 give different expressions compared to other studied miRNAs which may attribute to the adaptation to drought and salt stress and are good candidates for improving the crop by transgenic technology.

\section{References}

[1] Carrington, J.C. and Ambros, V. (2003) Role of MicroRNAs in Plant and Animal Development. Science, 301, 336-338. http://dx.doi.org/10.1126/science.1085242 
[2] Bartel, B. and Bartel, D.P. (2003) MicroRNAs: At the Root of Plant Development. Plant Physiology, 132, 709-717. http://dx.doi.org/10.1104/pp.103.023630

[3] Wang, Y. and Blelloch, R. (2009) Cell Cycle Regulation by MicroRNAs in Embryonic Stem Cells. Cancer Research, 69, 4093-4096. http://dx.doi.org/10.1158/0008-5472.CAN-09-0309

[4] Sunkar, R., Li, Y.F. and Jagadeeswaran, G. (2012) Functions of MicroRNAs in Plant Stress Responses. Trends in Plant Science, 17, 196-203. http://dx.doi.org/10.1016/j.tplants.2012.01.010

[5] Hamza, N.B., Elsadig, A.E., Elmunsor, I.I., Ibrahim, A.I.A. and Abuali, A.I. (2016) Assessment of Genetic Diversity in Grain Sorghum (Sorghum bicolor L. Moench) Genotypes under Drought Condition, Using Agro-Morphological Traits and Molecular Markers. International Journal of Plant Breeding and Genetics (Accepted, in Press).

[6] Li, W.X., Oono, Y., Zhu, J., He, X.J., Wu, J.M., Iida, K., Lu, X.Y., Cui, X., Jin, H. and Zhu, J.K. (2008) The Arabidopsis NFYA5 Transcription Factor Is Regulated Transcriptionally and Post-Transcriptionally to Promote Drought Resistance. Plant Cell, 20, 2238-2251. http://dx.doi.org/10.1105/tpc.108.059444

[7] Xia, K., Wang, R., Ou, X., Fang, Z., Tian, C., Duan, J., Wang, Y. and Zhang, M. (2012) OsTIR1 and OsAFB2 down Regulation via OsmiR393 over Expression Leads to More Tillers, Early Flowering and Less Tolerance to Salt and Drought in Rice. PLoS ONE, 7, 30-39.

[8] Zhang, Z.M., Lin, H.J., Shen, Y.O., Gao, J., Xiang, K., Liu, L., Ding, H.P., Yuan, G.S., Lan, H., Zhou, S.F., Zhao, M.J., Gao, S.B., Rong, T.Z. and Pan, G.T. (2012) Cloning and Characterization of miRNAs from Maize Seedling Roots under Low Phosphorus Stress. Molecular Biology Reports, 39, 8137-8146. http://dx.doi.org/10.1007/s11033-012-1661-5

[9] Doggett, H. (1988) Sorghum. 2nd Edition, Longmans Scientific and Technical, UK.

[10] Paterson, A.H., et al. (2009) The Sorghum Bicolor Genome and the Diversification of Grasses. Nature, 457, 551-556.

[11] Du, Z., Zhou, X., Ling, Y., Zhang, Z. and Su, Z. (2010) agriGO: A Goanalysis Toolkit for the Agricultural Community. Nucleic Acids Research, 38, W64-W70. http://dx.doi.org/10.1093/nar/gkq310

[12] Katiyar, A., Smita, S., Chinnusamy, V., Pandey, D.V. and Bansal, K.C. (2012) Identification of miRNAs in Sorghum by Using Bioinformatics Approach. Plant Signaling and Behavior, 7, 246-259. http://dx.doi.org/10.4161/psb.18914

[13] Zhang, W., Gao, S., Zhou, X., Chellappan, P., Chen, Z., Zhou, X., Zhang, X., Fromuth, N., Coutino, G., Coffey, M. and Jin, H. (2011) Bacteria-Responsive MicroRNAs Regulate Plant Innate Immunity by Modulating Plant Hormone Networks. Plant MolBiol, 75, 93-105. http://dx.doi.org/10.1007/s11103-010-9710-8

[14] Hamza, N.B., et al. (2016) MicroRNA Expression Profiles in Response to Drought Stress in Sorghum bicolor. Gene Expression Patterns (in Press). http://dx.doi.org/10.1016/j.gep.2016.01.001

[15] Kantar, M., Unver, T. and Budak, H. (2010) Regulation of Barley miRNAs upon Dehydration Stress Correlated with Target Gene Expression. Functional \& Integrative Genomics, 10, 493-507. http://dx.doi.org/10.1007/s10142-010-0181-4

[16] Liu, H.H., Tian, X., Li, Y.J., Wu, C.A. and Zheng, C.C. (2008) Microarray-Based Analysis of Stress-Regulated MicroRNAs in Arabidopsis thaliana. RNA, 14, 836-843. http://dx.doi.org/10.1261/rna.895308

[17] El-Sayed, H., El-Haddad, M. and Oleary, J.W. (1994) Effect of Salinity and K/Na Ratio of Irrigation Water on Growth and Solute Content of Atriplex amnicola and Sorghum bicolor. Irrigation Science, 14, 127-133.

[18] Nageshbabu, R., Jyothi, M.N., Sharadamma, N., Sarika, S., Rai, D.V. and Devaraj, V.R. (2013) Expression of miRNAs Regulates Growth and Development of French Bean (Phaseolus vulgaris) under Salt and Drought Stress Conditions. International Journal of Biological Sciences, 1, 52-56. 